\title{
Diagnostic Value of Magnetic Resonance Elastography Radiomics Analysis for the Assessment of Hepatic Fibrosis in Patients With Nonalcoholic Fatty Liver Disease
}

\section{Ki Choon Sim}

Korea University Anam Hospital, Korea University Medicine

Min Ju Kim ( $\square$ dr.minjukim@gmail.com )

Korea University Anam Hospital, Korea University Medicine

Yongwon Cho

Korea University Anam Hospital, Korea University Medicine Hyun Jin Kim

Korea University Anam Hospital, Korea University Medicine Beom Jin Park

Korea University Anam Hospital, Korea University Medicine

Deuk Jae Sung

Korea University Anam Hospital, Korea University Medicine

Yeo Eun Han

Korea University Anam Hospital, Korea University Medicine $\mathrm{Na}$ Yeon Han

Korea University Anam Hospital, Korea University Medicine Tae Hyung Kim

Korea University Ansan Hospital, Korea University Medicine Yoo-jin Lee

Korea University Anam Hospital, Korea University Medicine

\section{Research Article}

Keywords: Radiomics, Magnetic resonance imaging, Elastography, Nonalcoholic fatty liver disease, Liver fibrosis

Posted Date: August 14th, 2021

DOI: https://doi.org/10.21203/rs.3.rs-798827/v1 
License: (c) (i) This work is licensed under a Creative Commons Attribution 4.0 International License. Read Full License 


\section{Abstract}

Background: To investigate the diagnostic performance of radiomics analysis using magnetic resonance elastography (MRE) toward assessing hepatic fibrosis in patients with nonalcoholic fatty liver disease (NAFLD).

Methods: A total of 100 patients with suspected NAFLD were retrospectively enrolled. All patients underwent a liver parenchymal biopsy. MRE was performed using a 3.0-T scanner. Following threedimensional (3D) segmentation of MRE images, 834 radiomic features were analyzed using a commercial program. Radiologic features, such as median and mean values of two-dimensional (2D) or $3 \mathrm{D}$ regions of interest (ROIs) and variable clinical features, were analyzed. A random forest regressor was employed to extract important radiomic, radiological, and clinical features. A random forest classifier model was trained to use these features to classify the fibrosis stage. The area under the receiver operating characteristic curve (AUC) was evaluated using a classifier for fibrosis stage diagnosis.

Results: The pathological hepatic fibrosis stage was classified as low-grade fibrosis (stages F0-F1, $n=$ 82) or clinically significant fibrosis (stages $F 2-F 4, n=18$ ). Eight important features were extracted from radiomics analysis, with the two most important being wavelet-HHL gray level dependence matrix (GLDM)-dependence non-uniformity-normalized and wavelet-HHL GLDM-dependence entropy. The median value of the 2D ROI was identified as the most important radiologic feature. Platelet count was identified as an important clinical feature. The AUC of the classifier using radiomics was comparable to that of radiologic measures $(0.97 \pm 0.07$ vs. $0.96 \pm 0.06)$.

Conclusions: MRE radiomics analysis provides diagnostic performance comparable to conventional MRE analysis for the assessment of clinically significant hepatic fibrosis in patients with NAFLD.

\section{Background}

Chronic liver diseases are a common cause of morbidity and mortality worldwide, although specific diseases differ between regions [1]. The incidence of nonalcoholic fatty liver disease (NAFLD) has steadily increased in the general population [2,3]. Up to $40 \%$ of cases have been reported in the general population, and most patients are asymptomatic and only have simple steatosis. However, a small subset does have significant inflammation with or without fibrosis [3, 4]. Because serious complications, including hepatocellular carcinoma, occur in advanced fibrosis $[3,5]$, the accurate detection of significant hepatic fibrosis is of utmost importance.

Although percutaneous liver biopsy is the gold standard method for staging liver fibrosis, it has several drawbacks, including procedure-related complications and sampling errors [6, 7]. To overcome the limitations of liver parenchymal biopsies, various noninvasive quantitative methods, including ultrasound and magnetic resonance elastography (MRE), have been introduced and employed. MRE shows better organ coverage, reproducibility, diagnostic accuracy, sensitivity, and specificity as compared to other methods $[8,9]$. However, existing image analysis and post-processing MRE methods are limited by the 
exclusive use of mean signal intensity values through drawing of region of interest (ROI) [10]. To our knowledge, till date, there have been no studies that have attempted MRE analysis using other methods.

Radiomics analysis is a post-processing method that extracts and analyzes dozens to hundreds of features from various medical images. It extracts additional radiological information from features within images and spatial variations in pixel intensities that are undetectable by human perception [11-13]. Radiomics analysis of hepatic fibrosis using magnetic resonance imaging (MRI) has been examined in previous studies; however, these studies targeted patients with chronic viral hepatitis or used other sequences such as T2-weighted images or the hepatobiliary phase [14-16]. Radiomic analysis of hepatic fibrosis in patients with NAFLD has not been conducted thus far. We hypothesized that radiomic features extracted from MRE images would allow more accurate assessments of hepatic fibrosis than conventional ROI methods or the use of various clinical data. Accordingly, our study investigated the diagnostic performance of MRE radiomics for the assessment of clinically significant hepatic fibrosis in patients with NAFLD.

\section{Methods}

\section{Study population}

Approval for this retrospective investigation study was obtained from the Institutional review board (approval No. 2020AN0387), and the requirement for informed consent was waived. Between November 2017 to May 2020, 190 MREs were acquired. The inclusion criteria were as follows: (a) age $\geq 18$ years, (b) no known history of liver disease, (c) suspected NAFLD on screening based on ultrasound and laboratory studies, and (d) $\leq 60$ days between MRE and liver biopsy. The exclusion criteria were as follows: (a) history of alcohol consumption; (b) history of chronic liver diseases such as chronic hepatitis B or $C$ infection, autoimmune hepatitis, and primary sclerosing cholangitis; and (c) history of major liver surgery such as liver transplantation and hemihepatectomy. Based on these criteria, 100 consecutive patients were enrolled during the study period (Fig. 1).

Demographic, laboratory, and clinical features, including age, sex, weight, height, and blood test results (alanine aminotransferase [ALT], aspartate aminotransferase [AST], triglyceride, low-density lipoprotein, and platelet count) obtained within one month of MRE were evaluated. The body mass index and aspartate aminotransferase-to-platelet ratio index (APRI) were calculated.

\section{Reference standard for liver fibrosis}

Pathologic examination of the liver served as the reference standard for liver fibrosis. All patients underwent percutaneous liver biopsy using an 18-gauge needle with a $20-\mathrm{mm}$ penetration depth targeting segment $5 / 6$. Histology preparations from liver biopsies were retrospectively reviewed by one pathologist (7 years of experience) who was blinded to the clinical data and MRE results. The classification by Kleiner et al. [17] was used to grade and stage NAFLD. Fibrosis was staged between 0 and 4 as follows: F0, absence of fibrosis; F1, perisinusoidal or portal; F2, perisinusoidal and portal/periportal; F3, septal or 
bridging fibrosis; and F4, cirrhosis. The pathologic hepatic fibrosis stage was classified as mild fibrosis (F0 or F1) or clinically significant fibrosis (F2-F4) $[18,19]$.

\section{MRI examination}

MRI examinations were performed using 3.0-T scanners (Magnetom Skyra; Siemens Healthineers, Erlangen, Germany) with a 30-channel body coil. MRE was performed according to a previously described protocol $[20,21]$ using commercial hardware and software (Resoundant Inc., Rochester, MN, USA; Syngo MR E11, Siemens Healthineers). In the supine position, the passive acoustic driver was placed on the right chest wall and upper abdominal wall, with its center at the xiphoid process level. An elastic strap was used to secure it to the patient's body. MRE was performed using a phase-contrast gradient-recalled echo sequence, which applies motion-encoding gradients in synchronization with a $60-\mathrm{Hz}$ external shear wave induced in the abdomen. The total acquisition time was approximately $20 \mathrm{~s}$ per slice, and four contiguous slices were obtained for each patient. The acquisition parameters were as follows: axial plane, field of

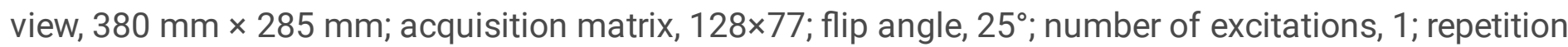
time ms/echo time ms, 50/17; and slice thickness and gap, $5 \mathrm{~mm}$. After the magnitude and phase images were obtained, an inversion algorithm installed in the MRI unit automatically processed raw data images to create several additional images and maps [22].

\section{MRE analysis processing}

Three-dimensional (3D) segmentation of MRE images was performed by three radiologists (two abdominal radiologists with 22 years $[n=21]$ and 10 years $[n=28]$ of clinical experience, respectively, and a 2-year resident $[n=51]$ ) using a commercial program, AVIEW (version 1.0.32.12, Coreline Soft, Seoul, Korea). As it was a time-consuming task, the three radiologists were randomly assigned patients to perform 3D segmentation. The segmentation was conducted on four contiguous grayscale elastograms with a 95\% confidence map (Fig. 2). All radiologists were trained by a software applicator to improve segmentation accuracy before they started the process. After completing the 3D segmentation, the mean and median values of liver fibrosis obtained from AVIEW were organized as 3D ROI values.

Using the grayscale elastogram with a 95\% confidence map, two circular ROls were defined per slice, and up to eight fibrosis values were obtained for each patient (Fig. 3). The ROI area was maintained at approximately $300-350 \mathrm{~mm}^{2}$. ROls were drawn at two separate sites or at one site while avoiding the edges of the liver [23]. All postprocessing was performed using a commercial workstation by a single abdominal radiologist who had 10 years of experience and was blinded to the clinicopathological data. The obtained liver fibrosis values were organized using mean and median values (2D ROI values). The 2D and 3D ROI values were used for the analysis of radiologic features.

\section{Radiomic feature extraction}

Based on MRE data with 3D segmentation applied, several hundreds of radiomic features were analyzed by an artificial intelligence research professor using PyRadiomics (version 3.0, PyRadiomics Community) 
(Fig. 4) [24]. The features included were related to shape, first-order statistical, second-order statistical (including so-called textural features such as the gray-level co-occurrence matrix, gray-level run-length matrix, gray-level dependence matrix, GLDM, gray level size zone matrix, and neighboring gray-tone difference matrix), and higher-order statistical using wavelet filters.

\section{Data analysis}

\section{Feature selection and classification method using machine learning}

Although many quantitative features (radiomics, radiologic, and clinical) can be extracted from medical datasets, these may be highly correlated with each other or simply considered as noise. Thus, it is important to reduce features to select a subset of specific features, enhance the performance, and minimize the computational cost. Among radiomics, radiologic, and clinical features, important features for predicting low-grade or clinically significant hepatic fibrosis in patients with NAFLD were selected using a random forest regressor in Python (Python Software Foundation, version 3.6) with the Scikit-learn package (https://github.com/scikit-learn/scikit-learn). A random forest classifier model [25] was trained to use these important features to classify the fibrosis stage. The 20 repeated 10fold stratified cross-validations verified the stability of the results. We evaluated the area under the receiver operating characteristic curve (AUC) and classifier accuracy. The classifier diagnosed the fibrosis stage based on radiomic, radiologic, or clinical features, or a combination of all features. Statistical differences in the AUC according to each classifier were compared using a machine learning model with Delong's test. $P$ values $<0.05$ were considered statistically significant.

\section{Conventional statistical analyses}

The demographic and clinical data in the low-grade fibrosis and clinically significant fibrosis groups were compared using the Mann-Whitney $U$ test, chi-squared test, or Fisher's exact test. The liver stiffness determined by the measurements was compared using a paired t-test. All statistical analyses were performed using SPSS Statistics for Windows (version 20.0; IBM Corp., NY, USA). $P$ values $<0.05$ were considered statistically significant.

\section{Results}

\section{Demographic and clinical characteristics}

As mentioned earlier, 100 patients were included in this study (Fig. 1). The mean interval between MRE and liver biopsies was 1.29 (range, 0-33) days. In 97 patients, the interval between MRE and liver biopsies was $<3$ days. Among the 100 patients, 16 were classified as having F0, 66 as having F1, five as having F2, six as having F3, and seven as having F4. Thus, the low-grade fibrosis group included 82 patients, while the clinically significant fibrosis group included 18 patients. Iron overload was suspected in only one patient in the MRE; however, this was not demonstrated in the pathology review. The demographic features of patients with NAFLD are shown in Table 1. 


\section{Liver stiffness measurement and MRE evaluation}

We aimed to obtain eight 2D ROls for each patient. However, because of some inappropriately acquired slices, one patient had five ROIs, two had six ROls, and four had seven ROIs. Liver stiffness values were organized according to the measurement method used in the two patient groups (Table 2). There was a significant difference in liver stiffness values between the two groups, regardless of the ROI measurement method $(P<0.001)$. There was no remarkable difference in 2D or 3D ROI values (approximately 2.5-2.7 $\mathrm{kPa}$ ) in the mild fibrosis group. However, in the significant fibrosis group, the 3D ROI value was lower than the 2D value (approximately $4.67-4.83 \mathrm{kPa}$ vs. $5.3 \mathrm{kPa}, P<0.001-0.002$ ).

\section{Comparison of diagnostic performances for classifying liver stiffness using machine learning}

The diagnostic performance for classifying liver stiffness and the important features extracted by each method are shown in Table 3.

\section{Classifying liver stiffness using radiomics}

The AUC and accuracy to discriminate between mild and significant fibrosis using radiomics were 0.97 and 0.94 , respectively. A total of eight radiomic features were extracted: wavelet-HHL GLDM-dependence non-uniformity normalized, wavelet-HHL GLDM dependence entropy, and original first-order median were determined to be the three most important features.

\section{Classifying liver stiffness using clinical features}

The AUC and accuracy of clinical features were 0.91 and 0.86 , respectively. A total of nine features were extracted; platelet count, APRI, and age were extracted as the three most important features.

\section{Classifying liver stiffness using radiologic ROI measures}

The AUC and accuracy of radiologic ROI measurements alone were 0.96 and 0.92 , respectively. The median of the 2D ROls was extracted as the most important feature.

\section{Classifying liver stiffness using a combination of radiomic, clinical, and radiologic measures}

The AUC and accuracy of all combinations of variable features were 0.98 and 0.95 , respectively. These values were higher than those for each of the features mentioned above. A total of 11 important features were extracted: wavelet-HHL GLDM dependence entropy, wavelet-HHL GLDM-dependence non-uniformity normalized, and the median of 2D ROls were extracted as the three most important features.

\section{Comparisons of AUCs of machine learning models with DeLong's test}

The AUC of the classifier determined using a combination of radiomics, radiologic measures, and clinical features ( $A U C=0.98$, accuracy $=0.95$ ) was slightly higher than that using radiomics $(A \cup C=0.97$, accuracy $=0.94)$ or radiologic measures $(A U C=0.96$, accuracy $=0.92)$ alone (Fig. 5). Clinical features 
alone showed the lowest diagnostic performance (AUC $=0.91$, accuracy $=0.86)$. There was a significant difference between usage of clinical features alone with that of the usage of combination of all features $(P=0.011)$, and use of radiomics alone $(P=0.039)$ (Table 4). However, there was no significant difference between using a combination of all features and radiomics alone $(P=0.960)$ or the combination of all features and radiologic measures $(P=0.254)$. Similarly, the virtually identical AUCs from radiologic measures and radiomics suggest that the added complexity of radiomics analysis does not afford a significant benefit $(P=0.694)$.

\section{Discussion}

Our study demonstrated that radiomics analysis of MRE (AUC $=0.97 \pm 0.07)$ could accurately differentiate clinically significant hepatic fibrosis (F2-F4) from low-grade fibrosis (F0 or F1) in patients with NAFLD. The AUC for MRE radiomics features $(0.97 \pm 0.07)$ was comparable with that for radiologic measures $(0.96 \pm 0.06)$. Therefore, the results of our study suggest that radiomics features of MRE analyzed using a machine learning approach can accurately stage liver fibrosis. The purpose of our study was to investigate the difference between MRE and ROI measurement, which is widely used as a gold standard, with radiomics analysis, having recently been in the spotlight. However, the AUC of MRE radiomics analysis showed no significant improvement compared to the conventional radiologic measures $(P=$ 0.694). Our results suggest that MRE radiomics analysis is not as effective as the conventional measurement method in terms of time and effort invested. In future, these disadvantages may be resolved by further development of software such as auto segmentation tools. This in turn would permit easy application of radiomics analysis in routine practice.

Previous studies using radiomics analysis and machine learning have attempted to differentiate the fibrosis stages of chronic liver disease using various MRI sequences. Lan et al. conducted a study to discriminate clinically significant fibrosis based on MRE [14]; however, because their study included patients with hepatitis $\mathrm{B} / \mathrm{C}$ and analyzed the AUC for each radiomic feature, directly comparison of the results of the two studies is not trivial. Other studies have attempted to predict liver stiffness by performing radiomics analysis using the hepatobiliary phase [15], or T1-weighted or T2-weighed images $[16,26]$ instead of MRE. Moreover, these three previous studies evaluated heterogeneous chronic liver diseases. Thus, it is challenging to directly compare the AUCs obtained in our study with these studies (AUC $=0.84-0.934$ ) because of differences in patient groups, MR equipment, and MRI sequences.

Considering each of the important features extracted in the MRE radiomics analysis and the combination of all features, we found two of them: GLDM-dependence non-uniformity-normalized and GLDMdependence entropy with a wavelet-HHL filter, to be more important than the others. Because the grayscale elastogram with a $95 \%$ confidence map reflects liver stiffness, a specific radiomic feature related to the gray level in the image is considered an important feature. Several radiomic features were presented by Lan et al. [14], who used MRE radiomics to diagnose clinically significant fibrosis in patients with chronic viral hepatitis; however, none of the radiomic features matched those identified in our study. A standard model for radiomic feature analysis has not been established, and the use of MR equipment 
with variable parameters due to vendor differences limits its clinical application. Thus, additional research is needed to identify the standard model and the most meaningful radiomic features.

In general, the shape features of radiomics analysis are reported to be of importance since they reflect the aggressiveness of several malignant tumors [27]. In radiologic imaging of diffuse liver disease, depending on the cause and stage of the disease, morphological changes distinguishable from normal liver are observed [28]; however, in the current study, shape features were not identified to be of importance in any analysis. There are several possible reasons for this observation. First, few patients progressed to advanced fibrosis, accompanied by morphological alterations. Second, subtle surface irregularities were not reflected during 3D segmentation. Finally, because the grayscale elastogram with a $95 \%$ confidence map does not accurately reflect the liver surface or whole volume, only four contiguous slices were obtained.

The median of the 2D ROls was extracted as the third most important feature in the combination of all features. Moreover, it was identified as the most important radiologic measure. The finding that the median of multiple small ROIs is more important than generally used radiologic measures, such as the mean ROI of a whole volume or mean of multiple small ROIs [10,23], is significant. Thus, MRE analysis can yield sufficient accuracy with only two appropriate ROIs per image, rather than using whole volume segmentation. Similarly, in liver stiffness measurements using FibroScan® (Echosens, France), which is widely used, the median value, and not the mean value of several repeated measurements, has been used [29].

Liver stiffness in the significant fibrosis group differed depending on whether it was a 2D or 3D ROI measurement; the overall liver stiffness was lower as per the 3D ROI measurement (Table 3). As the 2D $\mathrm{ROI}$ measurement was selected as a feature that is more important than the $3 \mathrm{D}$ measurement in the diagnostic performance analysis; it can be assumed that liver stiffness is generally lower with 3D measurements than with 2D ROI measurements (median $4.67 \mathrm{kPa} v \mathrm{~s}, 5.33 \mathrm{kPa}, P<0.001$ ). A possible reason could be that peripheral portions or areas showing relatively low stiffness in patients with advanced fibrosis were included in the 3D segmentation (Fig. 6). However, when 2D ROls were drawn for the same patient, the drawings were only in the central portion, which showed relatively high stiffness. Another possible reason may be that the overall stiffness was lower because of the included vessel area, considering that it is impossible to segment a whole volume excluding vessels in a $95 \%$ map with a low resolution. With manual ROI drawing, the possibility of liver stiffness masking by blood vessels may be lowered because blood vessels can be avoided following correlations with other imaging sequences. As shown in the present study, to avoid inclusion of peripheral liver portions, which can affect significant fibrosis diagnosis, FibroScan ${ }^{\circledR}$ recommends avoiding vessels and obtaining measurements at a depth of at least 25-65 $\mathrm{mm}$ from the skin [30].

Several demographics demonstrated significant differences between the mild and significant fibrosis groups (Table 1). Platelet count and AST showed the most significant differences, and there were significant differences in the APRI and AST/ALT ratio calculated using these two features. In both groups, 
ALT was elevated to a similar degree; however, in the mild fibrosis group, the AST value was relatively less elevated $(48.8 \pm 31.2 \mathrm{IU} / \mathrm{L}$ vs. $78.1 \pm 60.1 \mathrm{IU} / \mathrm{L}, P<0.05)$. A high AST/ALT ratio in patients with elevated AST and ALT levels is considered an important clinical finding suggestive of clinically significant fibrosis. Diabetes and old age were also found to differ between the groups, consistent with known risk factors for NAFLD [31]. In the analysis of clinical features using machine learning, platelet count, APRI, old age, and diabetes were identified as the top four important features (Table 3).

Our study had some limitations. First, this retrospective study might have had a selection bias. The pathologic fibrosis stage in our study population was not equivalent, and there were more patients in stages F0 and F1 than in stages F2-F4. This may indicate a spectrum bias and may have led to an overestimation of diagnostic performance. In addition, since this was a retrospective study, the reproducibility of the MRE examination could not be assessed. Second, the study population size was not large enough to be divided into training and validation sets to be used with machine learning. To overcome this limitation, we analyzed the results using 20 repeated 10 -fold stratified cross-validation. This method, as shown in previous studies [32-35], was the best way to increase the reliability of the evaluation using limited data. Third, external validation for machine learning was not performed, limiting the generalizability of our results. Finally, the inter- and intraobserver reliabilities of the 2D and 3D ROI measures could not be assessed. However, these shortcomings would have been overcome because 2D $\mathrm{ROI}$ drawings were performed up to eight times, and 3D segmentation was performed after sufficient training by a software applicator.

\section{Conclusions}

MRE radiomics analysis provides diagnostic performance comparable to that of conventional MRE analysis for the assessment of clinically significant hepatic fibrosis in patients with NAFLD.

\section{Abbreviations}

MRE, magnetic resonance elastography; NAFLD, nonalcoholic fatty liver disease; 3D, three-dimensional; 2D, two-dimensional; ROI(s), region-of-interest(s); AUC, area under the receiver operating characteristic curve; ALT, alanine aminotransferase; AST, aspartate aminotransferase; APRI, aspartate aminotransferase-to-platelet ratio index; GLDM, gray level dependence matrix.

\section{Declarations}

\section{Ethics approval and consent to participate}

The study was approved by the institutional review board of the Korea University Anam Hospital (approval No. 2020AN0387), we retrospectively enrolled 190 cases of magnetic resonance elastography of the liver from November 2017 to May 2020, and the requirement for informed consent was waived.

\section{Consent for publication}


Not applicable.

\section{Availability of data and materials}

The datasets used or analyzed during the current study are available from the corresponding author upon reasonable request.

\section{Competing interests}

The authors declare that they have no conflicts of interest.

\section{Funding}

Not applicable.

\section{Authors' contributions}

KCS proposed methods, performed the computations, and wrote the paper. MJK devised and supervised the project. MJK guaranteed the integrity of the entire study. KCS, MJK, YWC, BJP. DJS, YEH, NYH, THK, and YJL contributed to the interpretation of the data. KCS, YWC, and HJK contributed to the analysis and interpretation of the data. YWC solved some detailed problems in a deep learning experiment. All authors read and approved the final manuscript.

\section{Acknowledgments}

Not applicable.

\section{References}

1. Mokdad AA, Lopez AD, Shahraz S, Lozano R, Mokdad AH, Stanaway J, Murray CJ, Naghavi M. Liver cirrhosis mortality in 187 countries between 1980 and 2010: a systematic analysis. BMC Med. 2014;12:145.

2. Younossi ZM, Blissett D, Blissett R, Henry L, Stepanova M, Younossi Y, et al. The economic and clinical burden of nonalcoholic fatty liver disease in the United States and Europe. Hepatology. 2016;64:1577-86.

3. Stål P. Liver fibrosis in nonalcoholic fatty liver disease-diagnostic challenge with prognostic significance. World J Gastroenterol. 2015;21:11077-87.

4. Younossi ZM, Koenig AB, Abdelatif D, Fazel Y, Henry L, Wymer M. Global epidemiology of nonalcoholic fatty liver disease-meta-analytic assessment of prevalence, incidence, and outcomes. Hepatology. 2016;64:73-84.

5. Vernon G, Baranova A, Younossi ZM. Systematic review. The epidemiology and natural history of nonalcoholic fatty liver disease and nonalcoholic steatohepatitis in adults. Aliment Pharmacol Ther. 2011;34:274-85. 
6. Standish R, Cholongitas E, Dhillon A, Burroughs A, Dhillon AP. An appraisal of the histopathological assessment of liver fibrosis. Gut. 2006;55:569-78.

7. Bravo AA, Sheth SG, Chopra S. Liver biopsy. N Engl J Med. 2001;344:495-500.

8. Tang A, Cloutier G, Szeverenyi NM, Sirlin CB. Ultrasound elastography and MR elastography for assessing liver fibrosis: part 1, principles and techniques. AJR Am J Roentgenol. 2015;205:22-32.

9. Yoon JH, Lee JM, Joo I, Lee ES, Sohn JY, Jang SK, et al. Hepatic fibrosis: prospective comparison of MR elastography and US shear-wave elastography for evaluation. Radiology. 2014;273:772-82.

10. Bolster Jr B, Jin N, Kannengiesser S. MR Elastography-Pearls and Pitfalls. MAGMETOM Flash. 2018;71:19-23.

11. Gillies RJ, Kinahan PE, Hricak H. Radiomics: images are more than pictures, they are data. Radiology. 2016;278:563-77.

12. Lambin P, Leijenaar RTH, Deist TM, Peerlings J, de Jong EEC, van Timmeren J, et al. Radiomics: the bridge between medical imaging and personalized medicine. Nat Rev Clin Oncol. 2017;14:749-62.

13. Park HJ, Park B, Lee SS. Radiomics and Deep Learning: Hepatic Applications. Korean J Radiol. 2020;21:387-401.

14. Lan G-Y, Guo Y, Zhang X-Y, Cai X-L, Shi Y. Value of radiomic analysis of data from magnetic resonance elastography for diagnosing fibrosis stages in patients with hepatitis B/C. Chin $\mathrm{J}$ Acad Radiol. 2019;1:74-84.

15. Park HJ, Lee SS, Park B, Yun J, Sung YS, Shim WH, et al. Radiomics analysis of gadoxetic acidenhanced MRI for staging liver fibrosis. Radiology. 2019;290:380-7.

16. He L, Li H, Dudley JA, Maloney TC, Brady SL, Somasundaram E, et al. Machine learning prediction of liver stiffness using clinical and T2-weighted MRI radiomic data. AJR Am J Roentgenol. 2019;213:592-601.

17. Kleiner DE, Brunt EM, Van Natta M, Behling C, Contos MJ, Cummings OW, et al. Design and validation of a histological scoring system for nonalcoholic fatty liver disease. Hepatology. 2005;41:1313-21.

18. Hoodeshenas S, Yin M, Venkatesh SK. Magnetic resonance elastography of liver: current update. Top Magn Reson Imaging. 2018;27:319-33.

19. Venkatesh SK, Yin M, Takahashi N, Glockner JF, Talwalkar JA, Ehman RL. Non-invasive detection of liver fibrosis: MR imaging features vs. MR elastography. Abdom Imaging. 2015;40:766-75.

20. Venkatesh SK, Yin M, Ehman RL. Magnetic resonance elastography of liver: clinical applications. J Comput Assist Tomogr. 2013;37:887-96.

21. Loomba R, Wolfson T, Ang B, Hooker J, Behling C, Peterson M, et al. Magnetic resonance elastography predicts advanced fibrosis in patients with nonalcoholic fatty liver disease: a prospective study. Hepatology. 2014;60:1920-28.

22. Guglielmo FF, Venkatesh SK, Mitchell DG. Liver MR elastography technique and image interpretation: pearls and pitfalls. Radiographics. 2019;39:1983-2002. 
23. Chen J, Talwalkar JA, Yin M, Glaser KJ, Sanderson SO, Ehman RL. Early detection of nonalcoholic steatohepatitis in patients with nonalcoholic fatty liver disease by using MR elastography. Radiology. 2011;259:749-56.

24. van Griethuysen JJM, Fedorov A, Parmar C, Hosny A, Aucoin N, Narayan V, et al. Computational radiomics system to decode the radiographic phenotype. Cancer Res. 2017;77:e104-7.

25. Breiman L. Random forests. Mach Learn. 2001;45:5-32.

26. Schawkat K, Ciritsis A, von Ulmenstein S, Honcharova-Biletska H, Jüngst C, Weber A, et al. Diagnostic accuracy of texture analysis and machine learning for quantification of liver fibrosis in MRI: correlation with MR elastography and histopathology. Eur Radiol. 2020;30:4675-85.

27. Limkin EJ, Reuzé S, Carré A, Sun R, Schernberg A, Alexis A, et al. The complexity of tumor shape, spiculatedness, correlates with tumor radiomic shape features. Sci Rep. 2019;9:4329.

28. Wu J, Liu A, Cui J, Chen A, Song Q, Xie L. Radiomics-based classification of hepatocellular carcinoma and hepatic haemangioma on precontrast magnetic resonance images. BMC Med Imaging. 2019;19:23.

29. Kumar R, Rastogi A, Sharma MK, Bhatia V, Tyagi P, Sharma P, et al. Liver stiffness measurements in patients with different stages of nonalcoholic fatty liver disease: diagnostic performance and clinicopathological correlation. Dig Dis Sci. 2013;58:265-74.

30. Laurent S, Jennifer O, Cécile B, Céline F, Véronique M, Sebastian M. Non-invasive assessment of liver fibrosis by vibration-controlled transient elastography (Fibroscan $\left.{ }^{\circledR}\right)$. In: Takahashi H, editor. Liver biopsy. 6th ed. IntechOpen; 2011.

31. Duseja A, Chalasani N. Epidemiology and risk factors of nonalcoholic fatty liver disease (NAFLD). Hepatol Int. 2013;7 Suppl 2:755-64.

32. Suh $\mathrm{CH}$, Lee KH, Choi YJ, Chung SR, Baek JH, Lee JH, et al. Oropharyngeal squamous cell carcinoma: radiomic machine-learning classifiers from multiparametric MR images for determination of HPV infection status. Sci Rep. 2020;10:17525.

33. Wang XH, Long LH, Cui Y, Jia AY, Zhu XG, Wang HZ, et al. MRI-based radiomics model for preoperative prediction of 5-year survival in patients with hepatocellular carcinoma. $\mathrm{Br} \mathrm{J}$ Cancer. 2020;122:978-85.

34. Kniep HC, Madesta F, Schneider T, Hanning U, Schönfeld MH, Schön G, et al. Radiomics of brain MRI: Utility in prediction of metastatic tumor type. Radiology. 2019; 90:479-87.

35. Zhang Y, Oikonomou A, Wong A, Haider MA, Khalvati F. Radiomics-based prognosis analysis for nonsmall cell lung cancer. Sci Rep. 2017;7:46349.

\section{Tables}

Table 1. Demographic characteristics of patients with nonalcoholic fatty liver disease (NAFLD) 


\begin{tabular}{|c|c|c|c|}
\hline Patients & $\begin{array}{l}\text { Low-grade fibrosis }(n= \\
\text { 82) }\end{array}$ & $\begin{array}{l}\text { Clinically significant fibrosis }(n= \\
\text { 18) }\end{array}$ & $P$ value \\
\hline Sex (male:female) & $57: 25$ & $9: 9$ & 0.114 \\
\hline Hypertension (+ve) & $24(29.3 \%)$ & $8(44.4 \%)$ & 0.211 \\
\hline $\begin{array}{l}\text { Diabetes mellitus } \\
(+v e)\end{array}$ & $11(13.4 \%)$ & $11(61.1 \%)$ & $<0.001$ \\
\hline Age (years) & $45.4 \pm 13.3$ & $56.6 \pm 10.2$ & $<0.001$ \\
\hline BMI $\left(\mathrm{kg} / \mathrm{m}^{2}\right)$ & $28.6 \pm 5.2$ & $27.5 \pm 4.3$ & 0.501 \\
\hline AST (IU/L) & $48.8 \pm 31.2$ & $78.1 \pm 60.1$ & 0.015 \\
\hline ALT (IU/L) & $85.3 \pm 65.7$ & $71.6 \pm 45.7$ & 0.726 \\
\hline AST/ALT ratio & $0.7 \pm 0.4$ & $1.4 \pm 1.4$ & $<0.001$ \\
\hline Total TG (mg/dL) & $187.8 \pm 141.6$ & $184.3 \pm 89.5$ & 0.615 \\
\hline LDL & $121.7 \pm 30.6$ & $113.0 \pm 33.4$ & 0.24 \\
\hline $\begin{array}{l}\text { Platelet count }(x \\
\left.10^{3} / \mathrm{L}\right)\end{array}$ & $257.9 \pm 55.6$ & $171.0 \pm 35.5$ & $<0.001$ \\
\hline APRI & $0.50 \pm 0.34$ & $1.21 \pm 0.95$ & $<0.001$ \\
\hline NAS & $2.89 \pm 1.51$ & $3.60 \pm 1.77$ & 0.145 \\
\hline
\end{tabular}

$P$ values were derived using the independent t-test, Mann-Whitney $\mathrm{U}$ test, chi-squared test, or Fisher's exact test.

NAFLD, nonalcoholic fatty liver disease; BMI, body mass index; AST, aspartate transaminase; ALT, alanine aminotransferase; TG, triglyceride; LDL, low-density lipoprotein; APRI, aspartate aminotransferase-toplatelet ratio index; NAFLD, nonalcoholic fatty liver disease.

Table 2. Measurement of liver stiffness using magnetic resonance elastography in patients with nonalcoholic fatty liver disease 


\begin{tabular}{|llllll|}
\hline $\begin{array}{l}\text { Liver stiffness measurement } \\
\text { methods }\end{array}$ & \multicolumn{2}{l}{$\begin{array}{l}\text { Low-grade fibrosis, } \\
\mathbf{n = 8 2}\end{array}$} & $\begin{array}{l}\text { Clinically significant } \\
\text { fibrosis, } \mathbf{n}=18\end{array}$ & $P$ value \\
\cline { 2 - 5 } & $\begin{array}{l}\text { Mean } \pm \mathrm{SD} \\
(\mathrm{kPa})\end{array}$ & $\begin{array}{l}\text { Range } \\
(\mathrm{kPa})\end{array}$ & $\begin{array}{l}\text { Mean } \pm \mathrm{SD} \\
(\mathrm{kPa})\end{array}$ & $\begin{array}{l}\text { Range } \\
(\mathrm{kPa})\end{array}$ & \\
\hline 2D ROI measurement & & & & & \\
\hline Median & $2.53 \pm 0.62$ & $\begin{array}{l}1.56- \\
4.25\end{array}$ & $5.33 \pm 1.65$ & $3.26-9.20$ & $<0.001$ \\
\hline Mean & $2.58 \pm 0.63$ & $\begin{array}{l}1.59- \\
4.25\end{array}$ & $5.34 \pm 1.69$ & $3.06-9.29$ & $<0.001$ \\
\hline 3D ROI measurement & & & & & \\
\hline Median & $2.51 \pm 0.58$ & $\begin{array}{l}1.81- \\
4.13\end{array}$ & $4.67 \pm 1.23$ & $2.83-7.31$ & $<0.001$ \\
\hline Mean & $2.69 \pm 0.54$ & $\begin{array}{l}1.92- \\
4.36\end{array}$ & $4.83 \pm 1.24$ & $3.02-7.41$ & $<0.001$ \\
\hline
\end{tabular}

SD, standard deviation; ROI, region of interest; 2D, two-dimensional; 3D, three-dimensional

Table 3. Diagnostic performance for classifying liver stiffness and several important features of extracted for each method for in patients with nonalcoholic fatty liver disease 


\begin{tabular}{|c|c|c|c|c|}
\hline $\begin{array}{l}\text { Method of classifying liver } \\
\text { stiffness }\end{array}$ & AUC & Accuracy & Feature & $\begin{array}{l}\text { Feature } \\
\text { importance }\end{array}$ \\
\hline \multirow[t]{8}{*}{ Radiomics } & $\begin{array}{l}0.97 \\
\pm \\
0.07\end{array}$ & $\begin{array}{l}0.94 \pm \\
0.13\end{array}$ & $\begin{array}{l}\text { Wavelet-HHL GLDM } \\
\text { dependence non-uniformity } \\
\text { normalized }\end{array}$ & $\begin{array}{l}0.31005 \pm \\
0.02728\end{array}$ \\
\hline & & & $\begin{array}{l}\text { Wavelet-HHL GLDM } \\
\text { dependence entropy }\end{array}$ & $\begin{array}{l}0.24375 \pm \\
0.01775\end{array}$ \\
\hline & & & Original first order median & $\begin{array}{l}0.11797 \pm \\
0.01811\end{array}$ \\
\hline & & & $\begin{array}{l}\text { Wavelet-LLL first order } \\
\text { median }\end{array}$ & $\begin{array}{l}0.09658 \pm \\
0.02037\end{array}$ \\
\hline & & & $\begin{array}{l}\text { Wavelet-HHL GLDM } \\
\text { dependence variance }\end{array}$ & $\begin{array}{l}0.08890 \pm \\
0.01732\end{array}$ \\
\hline & & & $\begin{array}{l}\text { Wavelet-HHL GLCM joint } \\
\text { energy }\end{array}$ & $\begin{array}{l}0.06421 \pm \\
0.02168\end{array}$ \\
\hline & & & $\begin{array}{l}\text { Wavelet-HHH GLRML low } \\
\text { gray level run emphasis }\end{array}$ & $\begin{array}{l}0.05277 \pm \\
0.01339\end{array}$ \\
\hline & & & $\begin{array}{l}\text { Wavelet-HHL GLCM } \\
\text { maximum probability }\end{array}$ & $\begin{array}{l}0.02576 \pm \\
0.01374\end{array}$ \\
\hline \multirow[t]{9}{*}{ Clinical features } & $\begin{array}{l}0.91 \\
\pm \\
0.11\end{array}$ & $\begin{array}{l}0.86 \pm \\
0.20\end{array}$ & Platelet count & $\begin{array}{l}0.33412 \pm \\
0.03177\end{array}$ \\
\hline & & & APRI & $\begin{array}{l}0.13672 \pm \\
0.01692\end{array}$ \\
\hline & & & Age & $\begin{array}{l}0.11368 \pm \\
0.01720\end{array}$ \\
\hline & & & Diabetes mellitus & $\begin{array}{l}0.08113 \pm \\
0.01571\end{array}$ \\
\hline & & & Triglycerides & $\begin{array}{l}0.07980 \pm \\
0.00745\end{array}$ \\
\hline & & & Low-density lipoprotein & $\begin{array}{l}0.07809 \pm \\
0.01098\end{array}$ \\
\hline & & & Aspartate aminotransferase & $\begin{array}{l}0.07480 \pm \\
0.01358\end{array}$ \\
\hline & & & Alanine aminotransferase & $\begin{array}{l}0.05398 \pm \\
0.00850\end{array}$ \\
\hline & & & Body mass index & $\begin{array}{l}0.04768 \pm \\
0.00611\end{array}$ \\
\hline Radiologic measures & $\begin{array}{l}0.96 \\
\pm\end{array}$ & $\begin{array}{l}0.92 \pm \\
0.17\end{array}$ & Median of 2D ROls & $\begin{array}{l}0.30721 \pm \\
0.06659\end{array}$ \\
\hline
\end{tabular}




\begin{tabular}{|c|c|c|c|c|}
\hline & \multicolumn{4}{|l|}{0.06} \\
\hline & & & Mean of 3D ROls & $\begin{array}{l}0.25119 \pm \\
0.08035\end{array}$ \\
\hline & & & Mean of 2D ROls & $\begin{array}{l}0.24360 \pm \\
0.07967\end{array}$ \\
\hline & & & Median of 3D ROls & $\begin{array}{l}0.19800 \pm \\
0.06240\end{array}$ \\
\hline \multirow[t]{11}{*}{$\begin{array}{l}\text { Combination of radiomic, } \\
\text { clinical, and radiologic } \\
\text { measures }\end{array}$} & $\begin{array}{l}0.98 \\
\pm \\
0.04\end{array}$ & $\begin{array}{l}0.95 \pm \\
0.10\end{array}$ & $\begin{array}{l}\text { Wavelet-HHL GLDM } \\
\text { dependence entropy }\end{array}$ & $\begin{array}{l}0.17780 \pm \\
0.02365\end{array}$ \\
\hline & & & $\begin{array}{l}\text { Wavelet-HHL GLDM } \\
\text { dependence non-uniformity } \\
\text { normalized }\end{array}$ & $\begin{array}{l}0.15993 \pm \\
0.02046\end{array}$ \\
\hline & & & Median of 2D ROls & $\begin{array}{l}0.15427 \pm \\
0.02729\end{array}$ \\
\hline & & & Mean of 2D ROls & $\begin{array}{l}0.09536 \pm \\
0.01701\end{array}$ \\
\hline & & & Median of 3D ROls & $\begin{array}{l}0.09281 \pm \\
0.02347\end{array}$ \\
\hline & & & Mean of 3D ROls & $\begin{array}{l}0.08871 \pm \\
0.01810\end{array}$ \\
\hline & & & $\begin{array}{l}\text { Wavelet-LLL first order } \\
\text { median }\end{array}$ & $\begin{array}{l}0.04850 \pm \\
0.01805\end{array}$ \\
\hline & & & $\begin{array}{l}\text { Wavelet-HHL GLCM joint } \\
\text { energy }\end{array}$ & $\begin{array}{l}0.04764 \pm \\
0.02058\end{array}$ \\
\hline & & & Platelet count & $\begin{array}{l}0.04722 \pm \\
0.01561\end{array}$ \\
\hline & & & $\begin{array}{l}\text { Wavelet-HHL GLDM } \\
\text { dependence variance }\end{array}$ & $\begin{array}{l}0.04595 \pm \\
0.01581\end{array}$ \\
\hline & & & $\begin{array}{l}\text { Wavelet-HHH GLRML low } \\
\text { gray level run emphasis }\end{array}$ & $\begin{array}{l}0.04182 \pm \\
0.01147\end{array}$ \\
\hline
\end{tabular}

AUC, area under the receiver operating characteristic curve; ROIs, regions of interest; 2D, two-dimensional; 3D, three-dimensional; APRI, aspartate aminotransferase-to-platelet ratio index; GLCM, gray-level cooccurrence matrix; GLRML, gray-level run length matrix; GLDM, gray-level dependence matrix

Table 4. Pairwise comparisons of areas under the receiver operating characteristic curve using Delong's test 


\begin{tabular}{|c|c|c|c|}
\hline Variables & AUC & $95 \% \mathrm{Cl}$ & $P$ value \\
\hline $\begin{array}{l}\text { Combination of radiomic, radiologic measures, and clinical } \\
\text { features }\end{array}$ & $\begin{array}{l}0.98 \pm \\
0.04\end{array}$ & $0.951-1$ & 0.960 \\
\hline Radiomics & $\begin{array}{l}0.97 \pm \\
0.07\end{array}$ & $0.934-1$ & \\
\hline $\begin{array}{l}\text { Combination of radiomic, radiologic measures, and clinical } \\
\text { features }\end{array}$ & $\begin{array}{l}0.98 \pm \\
0.04\end{array}$ & $0.951-1$ & 0.254 \\
\hline Radiologic measures & $\begin{array}{l}0.96 \pm \\
0.06\end{array}$ & $0.928-1$ & \\
\hline $\begin{array}{l}\text { Combination of radiomic, radiologic measures, and clinical } \\
\text { features }\end{array}$ & $\begin{array}{l}0.98 \pm \\
0.04\end{array}$ & $0.951-1$ & 0.011 \\
\hline Clinical features & $\begin{array}{l}0.91 \pm \\
0.11\end{array}$ & $\begin{array}{l}0.811- \\
0.969\end{array}$ & \\
\hline Radiomics & $\begin{array}{l}0.97 \pm \\
0.07\end{array}$ & $0.934-1$ & 0.694 \\
\hline Radiologic measures & $\begin{array}{l}0.96 \pm \\
0.06\end{array}$ & $0.928-1$ & \\
\hline Radiomics & $\begin{array}{l}0.97 \pm \\
0.07\end{array}$ & $0.934-1$ & 0.039 \\
\hline Clinical features & $\begin{array}{l}0.91 \pm \\
0.11\end{array}$ & $\begin{array}{l}0.811- \\
0.969\end{array}$ & \\
\hline Radiologic measures & $\begin{array}{l}0.96 \pm \\
0.06\end{array}$ & $0.928-1$ & 0.062 \\
\hline Clinical features & $0.91 \pm$ & $\begin{array}{l}0.811- \\
0.969\end{array}$ & \\
\hline \multicolumn{4}{|c|}{ AUC, areas under the receiver operating characteristic curve; $\mathrm{Cl}$, confidence interval } \\
\hline
\end{tabular}




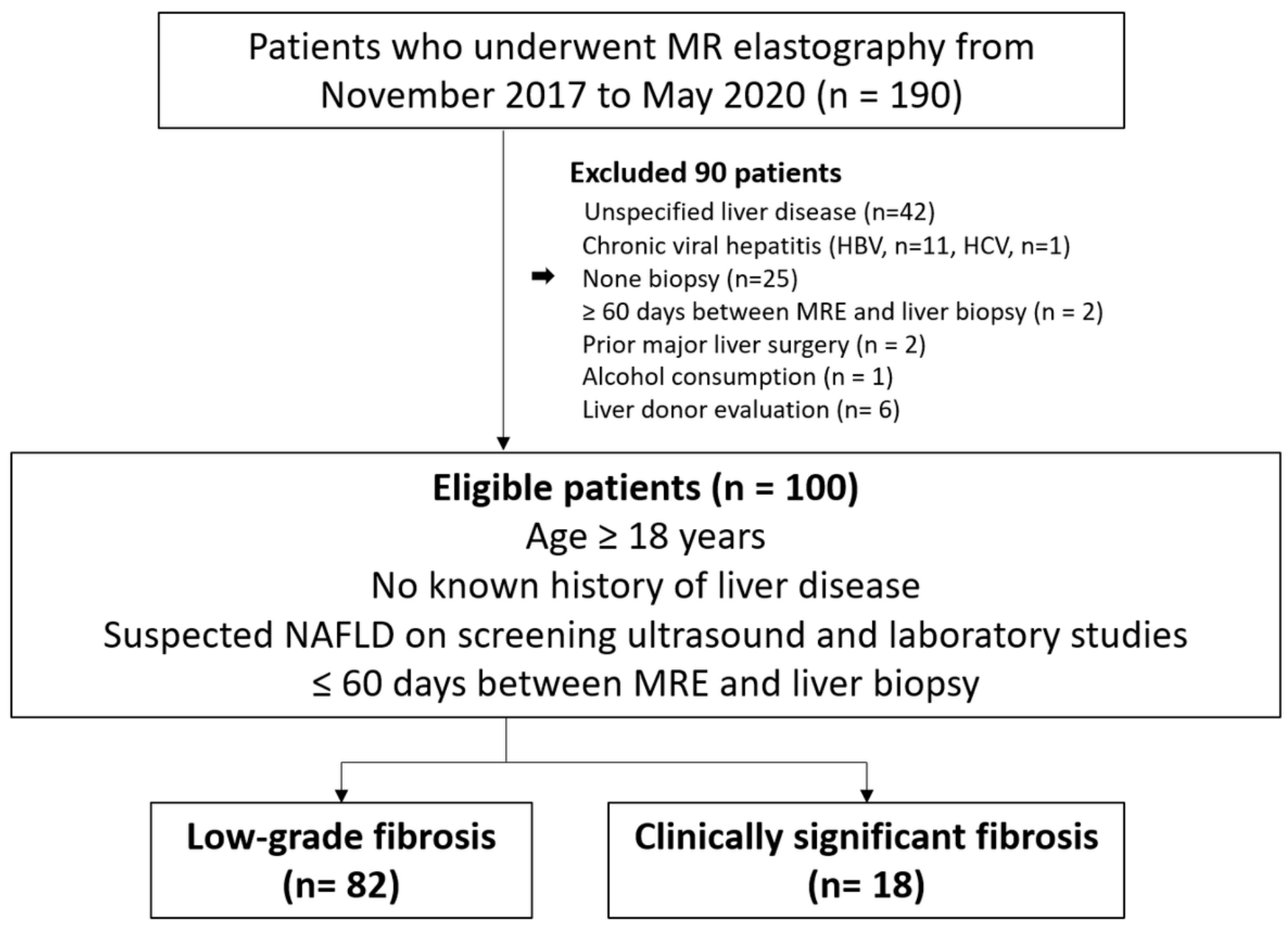

Figure 1

Flow chart depicting patient selection NAFLD, nonalcoholic fatty liver disease 


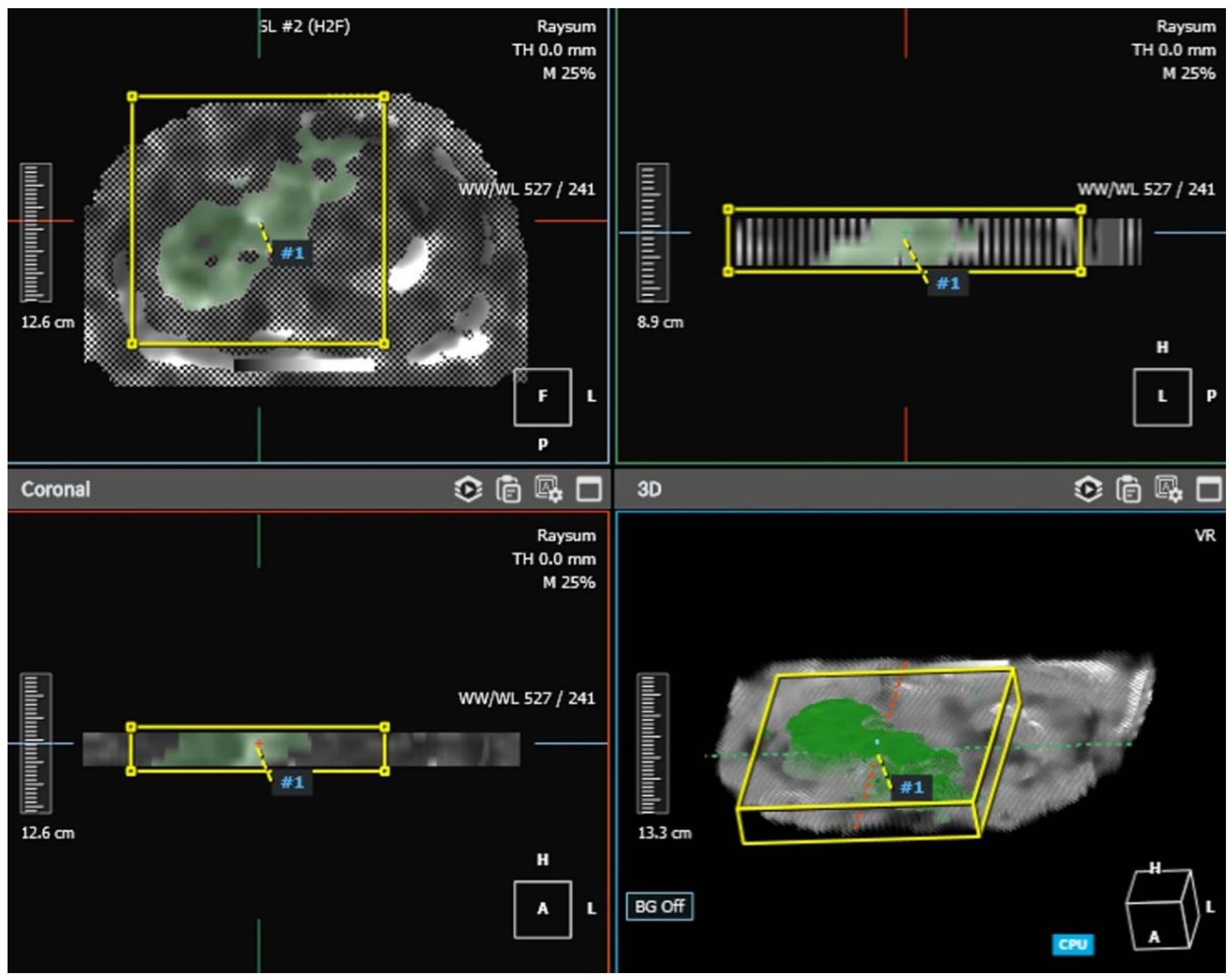

\section{Figure 2}

A 36-year-old man with NAFLD without hepatic fibrosis (stage F0; same as the patient in Fig. 1) The work screen shows the completion of the 3D segmentation of the grayscale elastogram with a 95\% confidence map using a commercial program. The median value of the whole volume ROI was $2.10 \mathrm{kPa}$, and the mean value was $2.24 \mathrm{kPa}$. NAFLD, nonalcoholic fatty liver disease; 3D, three-dimensional; ROI, region of interest. 


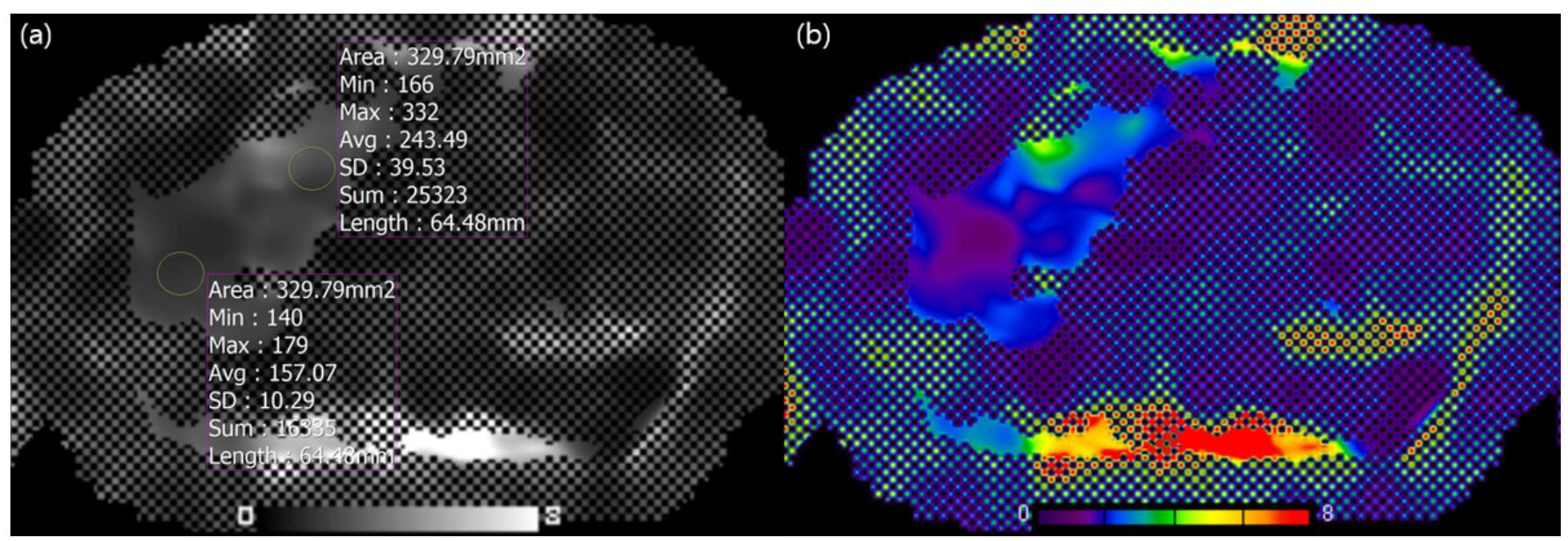

\section{Figure 3}

A 36-year-old man with NAFLD without hepatic fibrosis (Stage F0) (a) The axial grayscale elastogram with a 95\% confidence map shows an example of two separate circular ROls in one slice, avoiding the peripheral portion. The MRE measured liver stiffness at the two sites was approximately $1.57 \mathrm{kPa}$ and $2.43 \mathrm{kPa}$, respectively. The median value of multiple ROls was $1.75 \mathrm{kPa}$, and the mean value was 1.78 $\mathrm{kPa}$, which is appropriate for stage $\mathrm{F} 0$. (b) The color scale shows shear stiffness values ranging from 0 to $8 \mathrm{kPa}$, confirming the lack of significant fibrosis. NAFLD, nonalcoholic fatty liver disease; ROI, region of interest

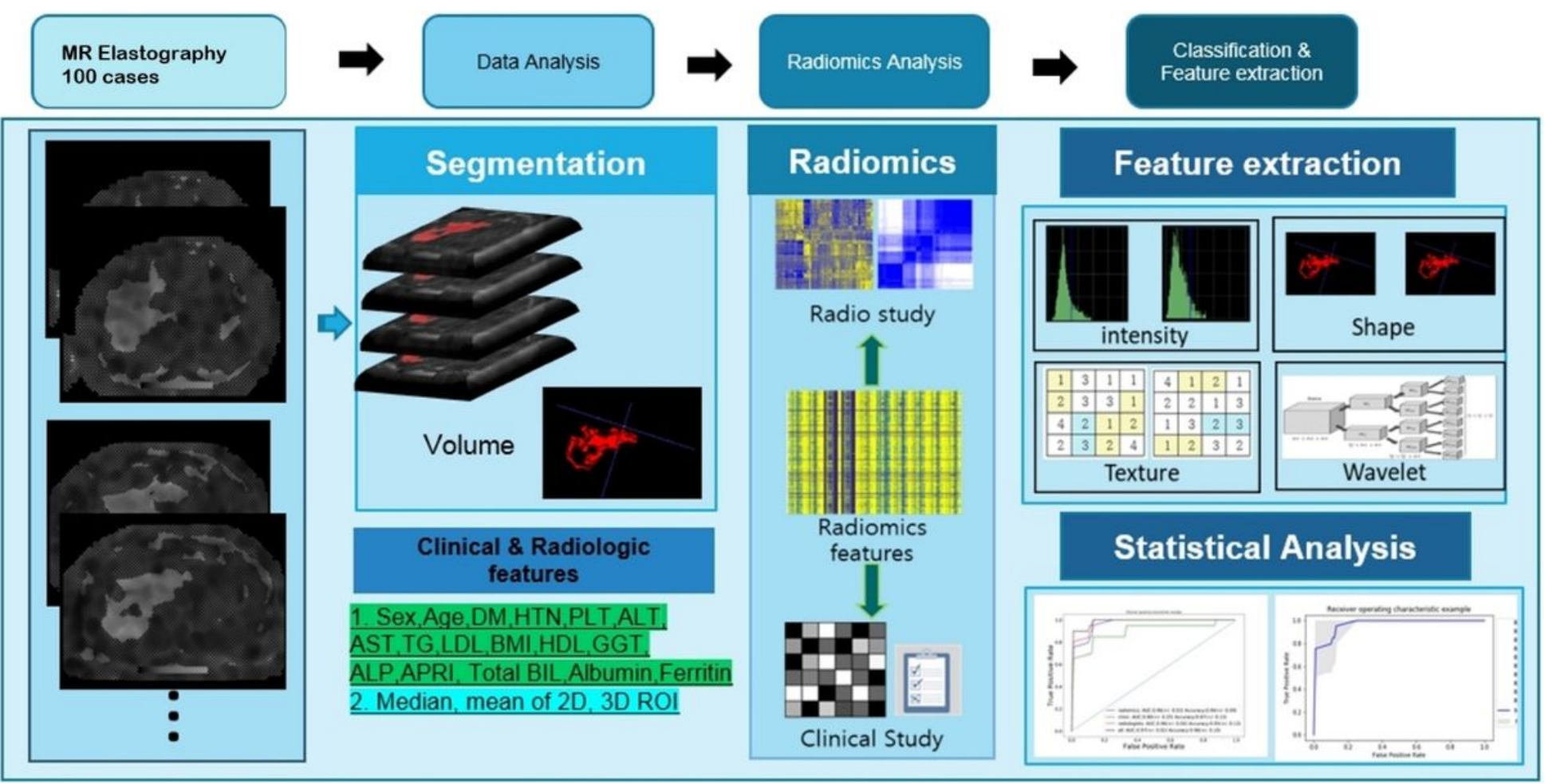

Figure 4 
Machine learning approach for classifying hepatic fibrosis according to MR elastography, clinical, and radiologic features 3D segmentation was performed using a grayscale elastogram with a $95 \%$ confidence map of the MR elastography. 2D and 3D ROI values from radiologic measurements and variable clinical data were also used in the analysis. Feature extraction was performed using 3D segmentation and clinical and radiologic data using PyRadiomics. A random forest regressor was used to extract the features based on their importance. The 20 repeated 10 -fold stratified cross-validations verified the stability of the results, and the area under the receiver operating characteristic curve of the classifier was evaluated. 2D, two-dimensional; 3D, three-dimensional; ROI, region of interest.

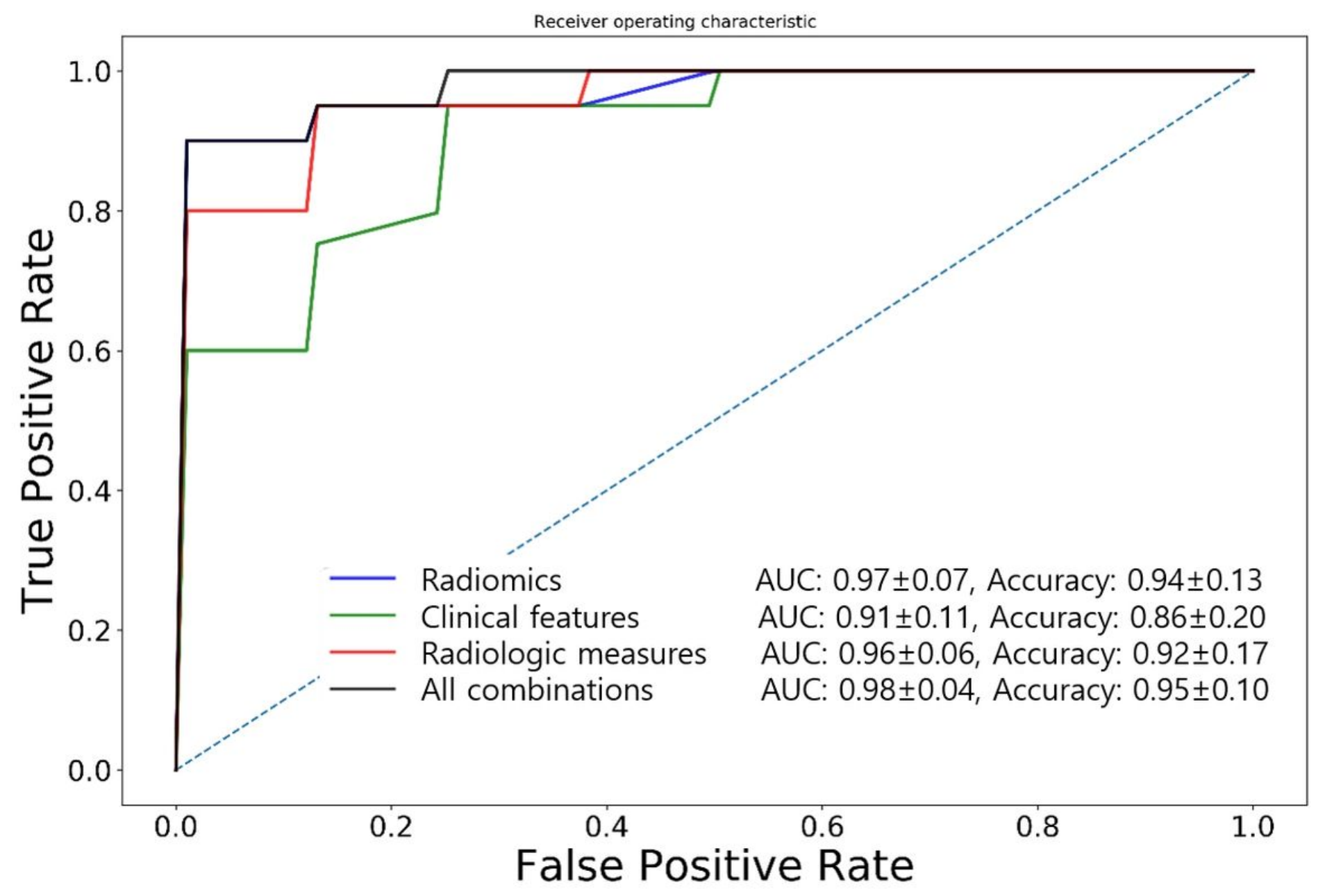

\section{Figure 5}

A random forest classifier model ROC curve This is used for diagnosing significant hepatic fibrosis using radiomics alone, clinical features alone, radiologic measures alone, and all features combined. Use of all combined features shows the highest AUC and accuracy. ROC, receiver operating characteristic; AUC, area under the ROC curve 


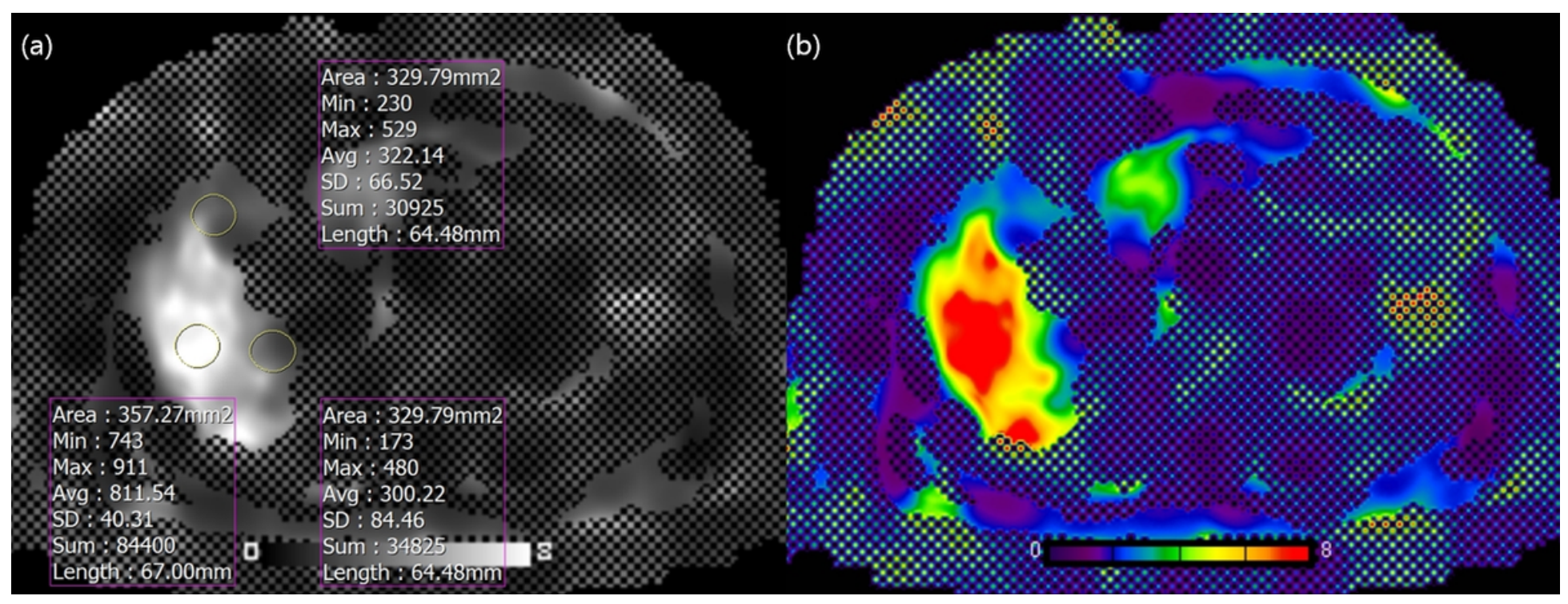

\section{Figure 6}

A 63-year-old woman with NAFLD with clinically significant hepatic fibrosis (Stage F3) The liver stiffness on MR elastography was as follows: 2D median value, $5.72 \mathrm{kPa}$; $2 \mathrm{D}$ mean value, $5.87 \mathrm{kPa}$; 3D median value, $5.14 \mathrm{kPa}$; and 3D mean value, $5.32 \mathrm{kPa}$. The axial grayscale elastogram with a $95 \%$ confidence map (a) and its corresponding RGB image (b) shows three separate circular ROls in one slice. The ROI of the most fibrotic area (asterisk) was $8.11 \mathrm{kPa}$, and that of the area just inside the grid was approximately 3-3.22 kPa. The peripheral portion or some area (blue area in RGB image) showing relatively low stiffness was included in the whole volume segmentation process, which may have lowered the overall stiffness value. NAFLD, nonalcoholic fatty liver disease; 2D, two-dimensional; 3D, three-dimensional; ROI, region of interest. 